\title{
Compact objects in conformal nonlinear electrodynamics
}

\author{
I. P. Denisova ${ }^{1}$, B. D. Garmaev², V. A. Sokolov ${ }^{2, a}$ \\ ${ }^{1}$ Moscow Aviation Institute (National Research University), Moscow Volokolamskoe Highway 4, 125993, Russia \\ 2 Physics Department, Moscow State University, Moscow 119991, Russia
}

Received: 29 March 2019 / Accepted: 11 June 2019 / Published online: 21 June 2019

(C) The Author(s) 2019

\begin{abstract}
In this paper we consider a special case of vacuum nonlinear electrodynamics with a stress-energy tensor conformal to the Maxwell theory. Distinctive features of this model are the absence of a dimensional parameter for the nonlinearity description and a very simple form of the dominant energy condition, which can easily be verified in an arbitrary pseudo-Riemannian space-time with the consequent constraints on the model parameters. In this paper we analyze some properties of astrophysical compact objects coupled to conformal vacuum nonlinear electrodynamics.
\end{abstract}

\section{Introduction}

Electromagnetic field theory, suggesting the possibility of nonlinear processes in vacuum due to the complicated dependence of the Lagrangian on the two electromagnetic field invariants, is usually called vacuum nonlinear electrodynamics. In spite of the extremely encouraging results in observing of vacuum birefringence in the strong magnetic field of a pulsar [1], predicted by some nonlinear models, the experimental status of vacuum electrodynamics remains unclear. The advances in the physics of intense electromagnetic fields and observational X-ray astronomy give hope for new experimental results in several projects such as extremely intensive laser facilities like ELI [2], XFEL [3], Apollon [4], XCELL [5], and orbital X-ray polarimeters like XIPE [6] and IXPE [7].

Early theoretical assumptions for vacuum electrodynamics nonlinearity were proposed in Born-Infeld [8] and Heisenberg-Euler [9] theories. Born-Infeld electrodynamics is a phenomenological model based on the assertion of finiteness of the electromagnetic field energy for a charged point-like particle. Later it was shown that this model arises in string theory as an effective action for an abelian vector field coupled to a virtual open Bose string [10]. As in Maxwell

a e-mail: sokolov.sev@inbox.ru electrodynamics, Born-Infeld theory induces a dual invariance [11] and displays no birefringence in vacuum [12]. An undoubted advantage of this theory is the strict and relatively simple form of the Lagrangian, which opens possibilities to find exact solutions. Nevertheless there are some disadvantages. The first one was noted by the authors immediately after establishing of the theory. The value of the electric field in the center of the point-like charge depends on the direction of approach. Resolving this problem leads to a modified Lagrangian [13]. The principle used for the BornInfeld Lagrangian construction turned out to be extremely productive and found application in other theoretical areas; for instance, it was implemented in several modifications of Born-Infeld gravity [14] and after supplementation by the AdS/CFT correspondence it was used for a description of holographic superconductors $[15,16]$.

Heisenberg-Euler theory [9] originates from quantum electrodynamics (QED) based on Maxwell theory and considers the radiative corrections to vacuum polarization in an external electromagnetic field, which leads to vacuum behavior as a continuous medium with a nonlinear features. This theory seems to be the one most profound and best justified, especially since some of its predictions have found experimental confirmation in a subcritical or perturbative regime. First of all, this refers to the electron anomalous magnetic moment correction, which still remains an example of the unprecedented correspondence between the theory and the experiment [17]. Some other QED predictions, such as Delbrück scattering [18], photon splitting [19], Lamb shift [20], and nonlinear Compton scattering [21], are also well established. There is no exact expression at all for the Heisenberg-Euler Lagrangian, and only a representation in the form of a series of loop corrections is available. This significantly complicates the analysis and often makes it impossible to obtain exact solutions. Moreover, due to the correspondence between Maxwell theory and nonlinear electrodynamics models, all of these models should lead to the Maxwell theory in the weak nonlinearity regime. In this case 
application of the quantization procedure and subsequent calculation of the loop corrections to any Lagrangian should lead for all of the models to the Heisenberg-Euler theory in the leading terms of Lagrangian expansion. Distinctive features of various nonlinear electrodynamics models after application of the quantization procedure will appear as modifications to the Heisenberg-Euler Lagrangian in terms of the high order of smallness. The effects coupled with such terms became significant only in a sufficiently nonlinear regime, with a strong electromagnetic field comparable to the SauterSchwinger limit. This regime of QED is poorly understood [22] and provides a new window for experimental and theoretical research. So confirmation of the Heisenberg-Euler theory predictions does not cancel the question as regards the choice of the theoretical model for nonlinear electrodynamics in classical field theory.

For this reason a set of new empirical models for nonlinear electrodynamics have been proposed. Some of them inherited the features of Born-Infeld electrodynamics; for instance, in [23] generalized Born-Infeld electrodynamics with two parameters was considered. This modification saves the finiteness of the electric field energy of the point-like charge, but it also leads to the prediction of vacuum birefringence. In this paper the exponential model of nonlinearity was also described. For both classes of electrodynamics the lowest-order modifications to the interaction energy of two point-like charges were calculated. As it follows from the results, for the noncommutative versions of the models, the interaction energy is ultraviolet finite.

Another remarkable model, with a logarithmic Lagrangian, was reviewed in [24]. Keeping the property of finite energy of a point charge in a flat space-time, this model leads to an exact solution of the field equations for which the event horizon coincides with the singularity point. Such objects are called black dots.

There are still other models with regular solutions for the electromagnetic field of the point-like source $[25,26]$. Among all empirical models, a special place is given to those that are inspired by astrophysics and cosmology [27,28], the development of which opened up an unusual theoretical view on the acceleration of the Universe due to nonlinear electromagnetic processes [29,30]. Moreover, charged regular black holes as a new class of compact astrophysical objects were predicted in [31,32]. As a rule, the choice of the Lagrangian for the model is heuristic and primarily based on the possibility to find an exact analytical solution for the case under consideration. At the same time, of great interest is the study of models grounded on more profound principles, one of which may be postulated as the maximal retention to the Maxwell theory properties and simultaneous prediction of the vacuum nonlinear response. In this paper we consider a general class of vacuum nonlinear electrodynamics with a zero trace for the stress-energy tensor. This condition is sufficient for the model to retain all group symmetries of the Maxwell theory, i.e. invariance under the Poincaré group, coordinate scaling and the conformal group [33]. Another distinctive feature of such models is the lack of a dimensional parameter describing the nonlinearity. For instance, in the Born-Infeld model, such a parameter is the value of the field strength in the center of the point-like charge, and in the Heisenberg-Euler theory this is the characteristic quantum induction. However, for the models under consideration in the paper, this parameter should be dimensionless, and, probably, it can be expressed as a combination of the fundamental constants. It should be noted that nowadays there are already some descriptions just for a particular models of nonlinear electrodynamics with a zero trace of the stress-energy tensor. The most vivid of them, see [34] and [35], are devoted to the charged black holes and their thermodynamics.

In this paper, we consider the most general form of the traceless nonlinear electrodynamics and describe some properties of the exact solutions for the compact astrophysical objects in such a model. This continues the series of papers, started by [36], in which vacuum birefringence for a general case of the traceless models was described.

The paper is organized as follows: In Sect. 2, we obtain the general form of the traceless nonlinear electrodynamics Lagrangian and discuss some of its features. In Sect. 3 we set fundamental restrictions on the Lagrangian. In Sect. 4 we obtain an analog of the Reissner-Nordström solution of the black hole with a dyon charge. Section 5 is devoted to the analog of the Vaidya-Bonnor solution and its features. In the last section we summarize our results. For convenience we will use geometerized units $(G=c=\hbar=1)$ and the metric signature $\{+,-,-,-\}$.

\section{Conformal vacuum nonlinear electrodynamics}

Let us consider the general form of the action for Lorentzinvariant vacuum nonlinear electrodynamics in a space-time with the metric tensor $g_{i k}$ :

$S_{m}=\int \sqrt{-g} \mathcal{L}\left(J_{2}, J_{4}\right) \mathrm{d}^{4} x$,

where the Lagrangian $\mathcal{L}$ is an arbitrary function of the electromagnetic field tensor $F_{i k}$ invariants $J_{2}=F_{i k} F^{k i}$ and $J_{4}=F_{i k} F^{k l} F_{l m} F^{m i}$, and $g$ is the determinant of the metric tensor. Varying the action by the metric $g^{i k}$ it is easy to derive a symmetric stress-energy tensor for the action (1):

$$
\begin{aligned}
T_{i k}=\frac{2}{\sqrt{-g}} \frac{\delta(\sqrt{-g} \mathcal{L})}{\delta g^{i k}}= & 4\left[\frac{\partial \mathcal{L}}{\partial J_{2}}+J_{2} \frac{\partial \mathcal{L}}{\partial J_{4}}\right] F_{i k}^{(2)} \\
& +\left[\left(2 J_{4}-J_{2}^{2}\right) \frac{\partial \mathcal{L}}{\partial J_{4}}-\mathcal{L}\right] g_{i k},
\end{aligned}
$$


whose trace is

$T=T_{i}^{i}=4\left[\frac{\partial \mathcal{L}}{\partial J_{2}} J_{2}+2 J_{4} \frac{\partial \mathcal{L}}{\partial J_{4}}-\mathcal{L}\right]$.

For brevity we introduce the notation for the second power of the electromagnetic field tensor of $F_{i k}^{(2)}=F_{i m} F_{k}^{m}$. It is well known that the Maxwell theory, which corresponds to the particular choice $\mathcal{L}=J_{2} / 16 \pi$, leads to a traceless stressenergy tensor. To retain this feature for vacuum nonlinear electrodynamics let us consider models with the action (1), for which the stress-energy tensor is conformal to Maxwell electrodynamics:

$T_{i k}=\Omega\left(J_{2}, J_{4}\right) T_{i k}^{M}=\frac{\Omega\left(J_{2}, J_{4}\right)}{4 \pi}\left\{F_{i k}^{(2)}-\frac{g_{i k}}{4} J_{2}\right\}$,

where $\Omega\left(J_{2}, J_{4}\right)$ is an arbitrary function of the electromagnetic invariants. It is easy to see that this requirement is fully similar to the traceless condition:

$J_{2} \frac{\partial \mathcal{L}}{\partial J_{2}}+2 J_{4} \frac{\partial \mathcal{L}}{\partial J_{4}}-\mathcal{L}=0$.

We will call the model with the Lagrangian satisfying Eq. (5) traceless or conformal nonlinear electrodynamics (CNED). Such a model name is justified, because the Lagrangians which satisfy (5) turn out to be invariant under the group of conformal-metric transformations $g_{i k} \rightarrow \tilde{g}_{i k}=$ $\lambda^{2}(x) g_{i k}$, where $\lambda$ is an arbitrary, scalar multiplier. A similar group symmetry is also inherent to Maxwell theory.

The CNED Lagrgangians have one more distinctive feature: the combination of CNED Lagrangians, under a certain condition, can also be a CNED Lagrangian. To obtain this condition let us consider the function $\mathcal{L}=\mathcal{L}\left(\mathcal{L}_{1}, \mathcal{L}_{2}\right)$ of the Lagrangians $\mathcal{L}_{1}$ and $\mathcal{L}_{2}$, which are a solutions of the traceless equation. After substitution of $\mathcal{L}$ to (5)

$$
\begin{aligned}
& \frac{\partial \mathcal{L}}{\partial \mathcal{L}_{1}}\left[J_{2} \frac{\partial \mathcal{L}_{1}}{\partial J_{2}}+2 J_{4} \frac{\partial \mathcal{L}_{1}}{\partial J_{4}}\right] \\
& +\frac{\partial \mathcal{L}}{\partial \mathcal{L}_{2}}\left[J_{2} \frac{\partial \mathcal{L}_{2}}{\partial J_{2}}+2 J_{4} \frac{\partial \mathcal{L}_{2}}{\partial J_{4}}\right]-\mathcal{L}\left(\mathcal{L}_{1}, \mathcal{L}_{2}\right)=0,
\end{aligned}
$$

and taking into account that $\mathcal{L}_{1}$ and $\mathcal{L}_{2}$ are also CNED Lagrangians, we get the equation, any solution of which will retain conformal features:

$\mathcal{L}_{1} \frac{\partial \mathcal{L}}{\partial \mathcal{L}_{1}}+\mathcal{L}_{2} \frac{\partial \mathcal{L}}{\partial \mathcal{L}_{2}}-\mathcal{L}\left(\mathcal{L}_{1}, \mathcal{L}_{2}\right)=0$.

The property noted above does not limit the possibilities of constructing CNED Lagrangians. For instance, to provide a correspondence to the Maxwell theory we choose the Lagrangian $\mathcal{L}_{1}=J_{2} / 16 \pi$. Another Lagrangian can be chosen in the form of the arbitrary function $\mathcal{L}_{2}=W\left(J_{2} / \sqrt{2 J_{4}}\right)$, which does not satisfy (5) and consequently is not conformally invariant. Nevertheless, the production of these Lagrangians will satisfy Eq. (5), and it is easy to verify that it will represent the most general form of CNED Lagrangian:

$\mathcal{L}=\mathcal{L}_{1} \mathcal{L}_{2}=\frac{J_{2}}{16 \pi} W\left(\frac{J_{2}}{\sqrt{2 J_{4}}}\right)=\frac{J_{2}}{16 \pi} W(z)$,

where the invariant ratio $z=J_{2} / \sqrt{2 J_{4}}$ varies from $z=-1$ for the purely magnetic field to $z=1$ for the purely electric field. Also it should be noted that $z$ is dimensionless, so there is no dimensional parameter at all to scale the model's nonlinearity. This fact leads to a very peculiar feature of the model coupled with correspondence to Maxwell theory. As a rule, models of nonlinear electrodynamics have a scale parameter with dimension of the field strength which defines the threshold for a substantially nonlinear regime. In the BornInfeld model such a parameter is the electric field strength in the center of the point-like charge, in Heisenberg-Euler electrodynamics this parameter is the characteristic quantum electrodynamic induction $B_{c}=m^{2} c^{3} / e \hbar=4.41 \cdot 10^{13} G$. At the weak field limit when, for instance, $|\mathbf{E}|,|\mathbf{B}| \ll B_{c}$, the model Lagrangian should correspond to Maxwell theory in the leading term of the expansion.

Contrary to this in the CNED model there is no dimensional parameter, so there is no way to scale the field and define the weak field limit. This peculiarity, possibly, will resolve after quantization of the $\mathrm{CNED}$ and radiation correction calculations. Then the quantum induction $B_{c}$ will play the role of a field scale of the model. However, it should be noted that when $W=1$ the CNED coincides with Maxwell electrodynamics. Therefore the correspondence principle in our case can be formulated in the form of the requirement that the unity should be the leading term of the expansion of the model function $W$ over the small dimensionless parameter, which is coupled to nonlinearity.

Despite the fact that for the Lagrangian (8) the traceless condition will be met with an arbitrary function $W$, there are some significant restrictions on this function, coming from fundamental principles.

\section{Fundamental restrictions}

The choice of the function $W(z)$ for each particular CNED model must fulfill fundamental principles, primarily the unitarity and causality conditions. The causality principle guarantees that the group velocity for the elementary electromagnetic excitations does not exceed the speed of light in the vacuum. The unitarity criterion provides the positive definiteness of the norm of every elementary excitation of the vacuum. The general constraints on the Lagrangian which are necessary for causality and unitarity are complicated and extremely difficult to analyze. In the same manner as in [37] we will consider the more particular case when the electric and magnetic fields meet the additional requirement $(\mathbf{E B})=0$ in a certain 
Lorentz frame. This corresponds to $z= \pm 1$. For the field configuration described in Ref. [37] a set of inequalities for the Lagrangian were obtained, which guarantee fulfillment of the causality and unitarity criteria:

$\frac{\partial \mathcal{L}}{\partial J_{2}} \geq 0, \quad \frac{\partial \mathcal{L}}{\partial J_{4}} \geq 0, \quad \frac{\partial \mathcal{L}}{\partial J_{2}}+J_{2} \frac{\partial \mathcal{L}}{\partial J_{4}} \geq 0$,

$\frac{\partial \mathcal{L}}{\partial J_{2}}+J_{2} \frac{\partial \mathcal{L}}{\partial J_{4}}+$

$\left[\frac{\partial^{2} \mathcal{L}}{\partial J_{2}^{2}}+2 J_{2} \frac{\partial^{2} \mathcal{L}}{\partial J_{2} \partial J_{4}}+J_{2}^{2} \frac{\partial^{2} \mathcal{L}}{\partial J_{4}^{2}}+\frac{\partial \mathcal{L}}{\partial J_{4}}\right] \geq 0$,

$\frac{\partial^{2} \mathcal{L}}{\partial J_{2}^{2}}+2 J_{2} \frac{\partial^{2} \mathcal{L}}{\partial J_{2} \partial J_{4}}+J_{2}^{2} \frac{\partial^{2} \mathcal{L}}{\partial J_{4}^{2}}+\frac{\partial \mathcal{L}}{\partial J_{4}} \geq 0$.

By taking into account that in general the CNED Lagrangian has the form (8), one can significantly simplify these inequalities, which finally leads to only two non-trivial constraints:

$W(z= \pm 1) \geq 0, \quad W^{\prime}(z= \pm 1) \leq 0$,

where the prime denotes the derivative with respect to $z$.

This restriction is not only because the Lagrangian must agree with the energy conditions. The class of conformal electrodynamics is special, because it has very simple restrictions following from the dominant energy condition [38]. This condition claims that every time-like observer will find the field energy density to be non-negative, and the energy flux to be a causal vector (time-like or null). These requirements ensure dominance of the energy density over the other components in the stress-energy tensor. The dominant energy condition leads to the inequalities

$T_{i k} a^{i} a^{k} \geq 0, \quad T_{k i} T^{i m} a_{m} a^{k} \geq 0$,

where $a^{k}$ is any arbitrary time-like vector pointing to the future. In general, after implementation to an arbitrary version of Lorentz-invariant nonlinear electrodynamics (1) the inequalities take a slightly cumbersome form:

$$
\begin{aligned}
T_{i k} a^{i} a^{k}= & 4\left\{\frac{\partial \mathcal{L}}{\partial J_{2}}+J_{2} \frac{\partial \mathcal{L}}{\partial J_{4}}\right\} F_{i k}^{(2)} a^{i} a^{k} \\
& +\left\{\left(2 J_{4}-J_{2}^{2}\right) \frac{\partial \mathcal{L}}{\partial J_{4}}-\mathcal{L}\right\} a_{k} a^{k} \geq 0, \\
T_{k i} T^{i m} a_{m} a^{k}= & 8\left\{\frac{\partial \mathcal{L}}{\partial J_{2}}+J_{2} \frac{\partial \mathcal{L}}{\partial J_{4}}\right\} \\
& \times\left\{J_{2} \frac{\partial \mathcal{L}}{\partial J_{2}}+2 J_{4} \frac{\partial \mathcal{L}}{\partial J_{4}}-\mathcal{L}\right\} F_{i k}^{(2)} a^{i} a^{k} \\
& +\left\{2\left[\frac{\partial \mathcal{L}}{\partial J_{2}}+J_{2} \frac{\partial \mathcal{L}}{\partial J_{4}}\right]^{2}\left(2 J_{4}-J_{2}^{2}\right)\right. \\
& \left.+\left[\left(2 J_{4}-J_{2}^{2}\right) \frac{\partial \mathcal{L}}{\partial J_{4}}-\mathcal{L}\right]^{2}\right\} a_{k} a^{k} \geq 0,
\end{aligned}
$$

however, they become much simpler in the context of CNED with the Lagrangian (8):

$$
\begin{aligned}
T_{i k} a^{i} a^{k}= & \frac{1}{4 \pi}\left[W+z\left(1-z^{2}\right) W^{\prime}\right] \\
& \times\left\{F_{i k}^{(2)} a^{i} a^{k}-\frac{J_{2}}{4} a_{k} a^{k}\right\} \geq 0, \\
T_{k i} T^{i m} a_{m} a^{k}= & \frac{J_{4}}{64 \pi^{2}}\left[W+z\left(1-z^{2}\right) W^{\prime}\right]^{2} \\
& \times\left\{1-\frac{z^{2}}{2}\right\} a_{k} a^{k} \geq 0 .
\end{aligned}
$$

The last inequality is always satisfied due to $|z| \leq 1$ and $J_{4} \geq 0$, while the last multiplier in (17) represents the energy condition for Maxwell theory and it's non negative. So the dominant energy condition for CNED will apply when

$W(z)+z\left(1-z^{2}\right) W^{\prime}(z) \geq 0$.

This inequality corresponds to the first one in (13) for purely electric or magnetic fields, when $z= \pm 1$. The restrictions obtained in the form of inequalities (13) and (19) allow one to find some constraints on the model parameters and they will be employed in the following sections for analysis of the consistency with the fundamental principles.

After discussion of the general properties and the model restrictions we proceed to address the description of an exact solution for compact objects in CNED.

\section{Reissner-Nordström black hole}

Primarily we describe a solution for the stationary black hole, with electric and magnetic charges in Einstein gravity with non-zero cosmological constant $\Lambda$. The action functional in this case can be represented in the form

$S=-\int \frac{R-2 \Lambda}{16 \pi} \sqrt{-g} \mathrm{~d}^{4} x+\int \mathcal{L} \sqrt{-g} \mathrm{~d}^{4} x$,

where $R$ is the scalar curvature, and $\mathcal{L}$ is the Lagrangian density (8). By varying the action, it is easy to derive the electromagnetic field and Einstein equations:

$R_{i k}-\frac{R}{2} g_{i k}+\Lambda g_{i k}=8 \pi T_{i k}$,

$\frac{1}{\sqrt{-g}} \frac{\partial \sqrt{-g} Q^{k n}}{\partial x^{n}}=-4 \pi j_{(e)}^{k}$,

$\frac{1}{\sqrt{-g}} \frac{\partial \sqrt{-g} * F^{k n}}{\partial x^{n}}=-4 \pi j_{(m)}^{k}$,

where $T_{i k}$ is the CNED stress-energy tensor (4), $* F^{k n}=$ $\mathrm{e}^{k n l m} F_{l m} / 2 \sqrt{-g}$ is the dual conjugate electromagnetic field tensor, $j_{(e)}^{k}$ and $j_{(m)}^{k}$ are the current density vectors for electric and magnetic charges, and the auxiliary tensor $Q^{k n}$ can be expressed in the form 
$Q^{k n}=W F^{k n}+z\left(F^{k n}-\frac{J_{2}}{J_{4}} F_{(3)}^{k n}\right) W^{\prime}$,

where the prime denotes the derivative of $W$ with the respect of its argument and $F_{(3)}^{k n}=F^{k l} F_{l m} F^{m n}$ is the third power of the field strength tensor. We consider the line element of the static spherically symmetric space-time:

$\mathrm{d} s^{2}=\mathrm{e}^{2 \alpha(r)} \mathrm{d} t^{2}-\mathrm{e}^{2 \beta(r)} \mathrm{d} r^{2}-r^{2}\left(\mathrm{~d} \theta^{2}+\sin ^{2} \theta \mathrm{d} \varphi^{2}\right)$,

and we also assume the most general form for the field strength tensor for a static point-like charge:

$F_{i k}=E(r)\left\{\delta_{i}^{0} \delta_{k}^{1}-\delta_{i}^{1} \delta_{k}^{0}\right\}-B(r) r^{2} \sin \theta\left\{\delta_{i}^{2} \delta_{k}^{3}-\delta_{i}^{3} \delta_{k}^{2}\right\}$,

where $E(r)$ and $B(r)$ are radial electric and magnetic fields. Under the chosen symmetries the invariants of the electromagnetic field and the dimensionless parameter $z=$ $J_{2} / \sqrt{2 J_{4}}$ take the form

$J_{2}=2\left[\mathrm{e}^{-2(\alpha+\beta)} E^{2}-B^{2}\right]$,

$J_{4}=2\left[\mathrm{e}^{-4(\alpha+\beta)} E^{4}+B^{4}\right]$,

$z=\frac{\mathrm{e}^{-2(\alpha+\beta)} E^{2}-B^{2}}{\left[e^{-4(\alpha+\beta)} E^{4}+B^{4}\right]^{1 / 2}}$.

As the point-like source is located at the coordinate center, the non-zero components of the current densities are

$j_{(e)}^{0}=\frac{Q_{c}}{4 \pi r^{2}} \mathrm{e}^{-(\alpha+\beta)} \delta(r), \quad j_{(m)}^{0}=\frac{Q_{t}}{4 \pi r^{2}} \delta(r)$,

where $Q_{c}$ is the electric charge and $Q_{t}$ is the topological charge of magnetic monopole. To obtain solutions for the electromagnetic field equations we consider the ansatz:

$E(r)=Q_{e} \mathrm{e}^{\alpha+\beta} / r^{2}, \quad B(r)=Q_{t} / r^{2}$,

where $Q_{e}$ is an integration constant coupled with the electric and magnetic charge of the black hole. As the field strength components have similar dependences on the coordinates, the argument $z$ takes a constant value and it can vary from $z=1$ for a purely electric field to $z=-1$ for purely magnetic field. By using the auxiliary expressions (26) and (23), it is easy to find that (28) is the solution of the electromagnetic field equations under the condition that the integration constant $Q_{e}$ and the charges $Q_{c}$ and $Q_{t}$ are coupled by the relation

$$
\begin{aligned}
& Q_{e}\left\{W(z)+\frac{z\left(1-z^{2}\right)}{2}\right. \\
& \left.\times\left[1+\frac{1-z^{2}}{1+\operatorname{sgn}(z) \sqrt{1-\left(1-z^{2}\right)^{2}}}\right] W^{\prime}(z)\right\}=Q_{c}, \\
& \text { where } z=\frac{Q_{e}^{2}-Q_{t}^{2}}{\left(Q_{e}^{4}+Q_{t}^{4}\right)^{1 / 2}} .
\end{aligned}
$$

A similar relation for the charges was obtained earlier in [35] for a particular choice of the electromagnetic field Lagrangian. Also it was found by the authors that for the chosen model $Q_{e}<Q_{c}$ when $Q_{t} \neq 0$. The appearance of two constants with the dimension of charge in the description of the point source field can be associated with the possible difference between the inertial and gravitational masses for a point-like particle. As the constant $Q_{e}$ is contained in the expression of the electric field strength, it can be called "force-charge", in contrast to the constant $Q_{c}$, which is the multiplier in the source density, so it can be called "sourcecharge" or Coulomb charge. At the same time, it is interesting to find the condition under which the source-charge and the force-charge coincide, $Q_{e}=Q_{c}$, in the presence of a topological charge $Q_{t} \neq 0$. The claim is satisfied when the expression in the curly brackets in (29) is equal to unity, which can be done by choosing

$W(z)=1+\frac{c_{1}}{z} \sqrt{1-\operatorname{sgn}(z) \sqrt{1-\left(1-z^{2}\right)^{2}}}$,

where $c_{1}$ is an arbitrary dimensionless constant. It is easy to verify that the restrictions from the causality and unitarity (13) now can be expressed in the form $W^{\prime}(z= \pm 1)=$ $-\sqrt{2} c_{1} \leq 0$, so the constant $c_{1} \geq 0$ should be positive. At the same time, the dominant energy condition (19) leads to the inequality

$$
\begin{aligned}
& W+z\left(1-z^{2}\right) W^{\prime} \\
& =1-\frac{c_{1} z \sqrt{1-\operatorname{sgn}(z) \sqrt{1-\left(1-z^{2}\right)^{2}}}}{\operatorname{sgn}(z) \sqrt{1-\left(1-z^{2}\right)^{2}}} \geq 0,
\end{aligned}
$$

the second term in which is finite, takes the minimal value $-c_{1} \sqrt{2}$ at $z=-1$ and increases monotonically up to the zero at $z=1$. So the dominant energy condition for the CNED model described will be satisfied when $0 \leq c_{1} \leq$ $\sqrt{2}$. From the experimental data for the vacuum nonlinear electrodynamics effects it seems that the nonlinearity is a small correction to the Maxwell electrodynamics, so $c_{1} \ll 1$ and implementation of inequality (31) is reliably ensured.

The other distinctive case of CNED take place when $Q_{c}=$ 0 and $Q_{e} \neq 0$, which means that the topological charge $Q_{c}$ will be the source both for the electric and magnetic fields. In this case the Lagrangian should be expressed with the function $W(z)$ from (30) minus unity.

However, from the previous consideration, it is obvious that this model contradicts the causality and unitarity conditions (13) and it is unlikely for it to be related to the real world.

We proceed to a solution of the Einstein equations (21), which for static space-time with the line element (24) have only two non-trivial and independent equations:

$$
\begin{aligned}
& e^{-2 \beta}\left[\frac{2 \beta^{\prime}}{r}-\frac{1}{r^{2}}\right]+\frac{1}{r^{2}}+\Lambda=8 \pi T_{0 \cdot}^{.0}, \\
& -e^{-2 \beta}\left[\frac{2 \alpha^{\prime}}{r}+\frac{1}{r^{2}}\right]+\frac{1}{r^{2}}+\Lambda=8 \pi T_{1 \cdot}^{\cdot 1},
\end{aligned}
$$


where the non-zero components of the stress-energy tensor for the chosen electromagnetic field configuration are

$$
\begin{aligned}
T_{0 \cdot}^{\cdot 0} & =T_{1 \cdot}^{\cdot 1}=-T_{2 \cdot}^{\cdot 2}=-T_{3 \cdot}^{\cdot 3} \\
& =\frac{Q_{e}^{2}+Q_{t}^{2}}{8 \pi r^{4}}\left[W(z)+z\left(1-z^{2}\right) W^{\prime}(z)\right],
\end{aligned}
$$

and the prime denotes the derivative with respect to the correspondent argument. Subtracting the second equation (32) from the first one, we obtain the condition $\alpha+\beta=f(t)$, where the arbitrary function $f$ can be taken equal to zero after the choice of the time scale, so the solution for both equations can be written in the following form:

$$
g_{00}(r)=\mathrm{e}^{2 \alpha(r)}=\mathrm{e}^{-2 \beta(r)}=1-\frac{2 M}{r}+\frac{\mathcal{K}}{r^{2}}+\frac{1}{3} \Lambda r^{2},
$$

where the integration constants were chosen to obtain the asymptotic limit to the solution in Einstein-Maxwell theory. Therefore, $M$ is the black hole mass and, for brevity, as in [35], we use the notation

$\mathcal{K}=\left\{Q_{e}^{2}+Q_{t}^{2}\right\}\left[W(z)+z\left(1-z^{2}\right) W^{\prime}(z)\right]$.

The solution obtained corresponds to the Reissiner-Nordström black hole in CNED and differs from the similar one obtained in [35] by the more general form of the parameter $\mathcal{K}$ coupled to an arbitrary CNED Lagrangian; thus the analysis of the black hole thermodynamics performed in [35] can be completely applied to (34). In this paper the authors distinguish thee different classes of black holes. The first one corresponds to the case when $\mathcal{K}>\Lambda / 12$ and was called fast black holes. Phase transitions are absent for this black hole configuration. For the second class, called slow black holes, $0<\mathcal{K}<\Lambda / 12$ and there are two phase transitions. These two types of black holes have their analogs in EinsteinMaxwell theory. The third class, inverse black holes, corresponds to $\mathcal{K}<0$ and possesses solely one phase transition. This type of black hole is typical for conformal-invariant electrodynamics, and we meet it in the more special case of the inverse electrodynamics model proposed in [35]. However, it should be noted that the authors did not consider fundamental restrictions on the model parameters. In particular, the existence of the inverse black holes class contradicts the dominant energy condition (19), the consequence of which is the restriction $\mathcal{K} \geq 0$. A global violation of this condition makes the possibility of such black holes very uncertain.

Let us turn to the other exact solution for the compact astrophysical object in conformal nonlinear electrodynamics.

\section{Vaidya-Bonnor radiating solution}

Let us consider the solution of the Einstein-CNED equations describing the emission of a charged null fluid from a spherically symmetric star with electric and magnetic charges. This solution will be an extension of Vaidya-Bonnor [39] metric to an arbitrary type of conformal nonlinear electrodynamics. To obtain the solution, as is traditional, we assume the line element in Eddington-Finkelstein coordinates to be of the form

$\mathrm{d} s^{2}=G(u, r) \mathrm{d} u^{2}+2 \mathrm{~d} u \mathrm{~d} r-r^{2}\left(\mathrm{~d} \theta^{2}+\sin ^{2} \theta \mathrm{d} \varphi^{2}\right)$,

where $G$ is the metric function and $u$ is the retarded time.

As in Sect. 4, we represent the electromagnetic field tensor in the form (25) and assume the electromagnetic field strengths to be $B=Q_{t} / r^{2}, E=Q_{e} / r^{2}$. However, unlike the Reissner-Nordström solution, the topological charge $Q_{t}=Q_{t}(u)$, the source-charge $Q_{c}=Q_{c}(u)$, the forcecharge $Q_{e}=Q_{e}(u)$ and the star mass $M=M(u)$ are arbitrary functions of the retarded time. It is easy to verify that the electromagnetic field satisfies Eq. (22) with the same form of the $Q^{k n}$ tensor (23) and the following form of the current densities for the electric and magnetic charges:

$$
\begin{aligned}
J_{(e)}^{k} & =\frac{1}{4 \pi r^{2}}\left[Q_{c} \delta(r) \delta_{0}^{k}-\dot{Q}_{c}(u) \delta_{1}^{k}\right], \\
J_{(m)}^{k} & =\frac{1}{4 \pi r^{2}}\left[Q_{t} \delta(r) \delta_{0}^{k}-\dot{Q}_{t}(u) \delta_{1}^{k}\right],
\end{aligned}
$$

where the dot denotes the derivative with respect to the retarded time, $\delta_{i}^{k}$ is the Kronecker symbol and the sourcecharge $Q_{c}(u)$ is coupled to the force-charge $Q_{e}(u)$ by the same relation as (29). To find the modified Vaidya-Bonnor metric it is necessary to include the stress-energy tensor of the null fluid in the Einstein equations:

$R_{i k}-\frac{R}{2} g_{i k}+\Lambda g_{i k}=8 \pi\left(T_{i k}+V_{i} V_{k}\right)$

where $V_{i}$ is the null-fluid current vector, for which $g_{i k} V^{i} V^{k}$ $=0$. Finally, for completeness we also assume the Lambdaterm to be a function varying with the retarded time $\Lambda=$ $\Lambda(u)$. Einstein's equations in this case take the form

$$
\begin{aligned}
& \frac{\partial}{\partial r}[r G(u, r)]=1+r^{2} \Lambda(u)-8 \pi r^{2}\left(T_{0 \cdot}^{\cdot 0}+V_{0} V^{0}\right), \\
& \frac{1}{r} \frac{\partial G(u, r)}{\partial u}=8 \pi V_{0} V^{1}
\end{aligned}
$$

where the electromagnetic field stress-energy tensor components can be expressed from (33). As in the original paper by Vaidya and Bonnor [39] we assume the null-fluid current density to be radial and represent it with the scalar $N=N(u, r)$ in the form $V^{i}=N \delta_{1}^{i}$, so the solution of (39) reads

$$
\begin{aligned}
& G(u, r)=1-\frac{2 M(u)}{r}+\frac{\mathcal{K}(u)}{r^{2}}+\frac{1}{3} \Lambda(u) r^{2}, \\
& N^{2}=\frac{3 \dot{\mathcal{K}}-6 \dot{M} r+\dot{\Lambda} r^{4}}{24 \pi r^{3}}
\end{aligned}
$$




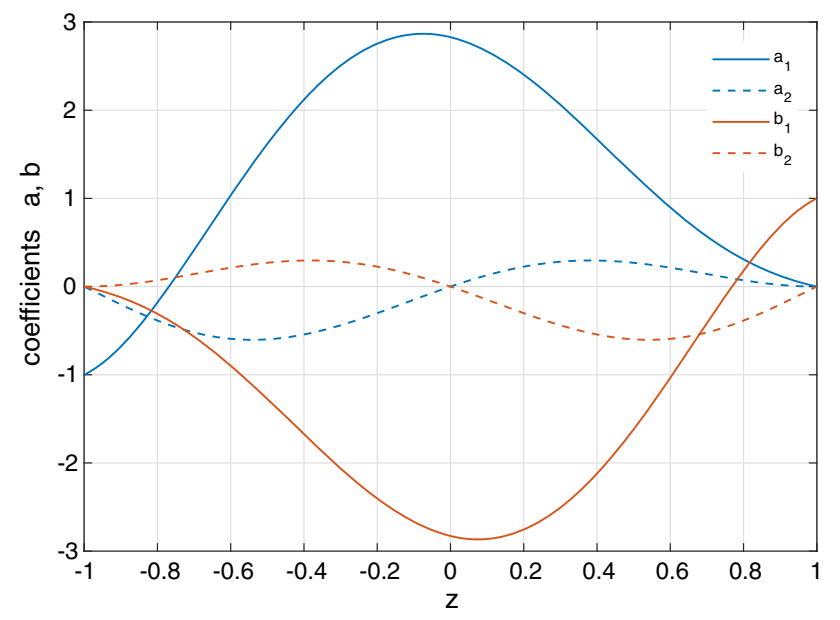

Fig. 1 Coefficients $a$ and $b$ as functions of $z$

where the expression for $\mathcal{K}$ coincides with (35) and its derivative is

$$
\begin{aligned}
\dot{\mathcal{K}}= & \frac{d}{d u}\left\{\left[Q_{e}^{2}(u)+Q_{t}^{2}(u)\right] \times\left[W(z)+z\left(1-z^{2}\right) W^{\prime}(z)\right]\right\} \\
= & 2 Q_{e} \dot{Q}_{e}\left\{W+a_{1} W^{\prime}+a_{2} W^{\prime \prime}\right\} \\
& +2 Q_{t} \dot{Q}_{t}\left\{W+b_{1} W^{\prime}+b_{2} W^{\prime \prime}\right\}
\end{aligned}
$$

with the auxiliary notations for the coefficients, introduced for brevity:

$$
\begin{aligned}
& a_{1}=\left(1-z^{2}\right)\left[z+\frac{\left(2-z^{2}\right)\left(2-3 z^{2}\right)}{\sqrt{2+2 \operatorname{sgn}(z) \sqrt{1-\left(1-z^{2}\right)^{2}}}}\right], \\
& a_{2}=\frac{z\left(2-z^{2}\right)\left(1-z^{2}\right)^{2}}{\sqrt{2+2 \operatorname{sgn}(z) \sqrt{1-\left(1-z^{2}\right)^{2}}}}, \\
& b_{1}=\left(1-z^{2}\right)\left[z-\frac{\left(2-z^{2}\right)\left(2-3 z^{2}\right)}{\sqrt{2-2 \operatorname{sgn}(z) \sqrt{1-\left(1-z^{2}\right)^{2}}}}\right], \\
& b_{2}=-\frac{z\left(2-z^{2}\right)\left(1-z^{2}\right)^{2}}{\sqrt{2-2 \operatorname{sgn}(z) \sqrt{1-\left(1-z^{2}\right)^{2}}}} .
\end{aligned}
$$

The dependence of the coefficients $a$ and $b$ on $z$ is represented on Fig. 1. It should be noted that $a_{2}=0, b_{2}=0$ for a purely magnetic and electric field when $z= \pm 1$. These coefficients are also equal to zero when $z=0$ at $Q_{e}= \pm Q_{t}$. For the listed cases only the values of $W$ and $W^{\prime}$, which are restricted by the causality and unitarity conditions (13), will handle $\dot{\mathcal{K}}$, the expressions for which in these particular cases can be found in Table 1.

To ensure that $N^{2}$ is not negative for an arbitrary distance to the star center, it is necessary to require that the star mass decrease with the retarded time $\dot{M} \leq 0$, the Lambda-term should vary with $\dot{A} \geq 0$, following cosmological dynamics, while $\mathcal{K}$ should increase due to ionization processes in the star and radiation of the charged fluid. The condition $N^{2} \geq 0$ should be verified for each particular instance of the model function $W$, however, as it follows from (41) it will be certainly fulfilled when

$$
\frac{\mathrm{d}}{\mathrm{d} u}\left[W(z)+z\left(1-z^{2}\right) W^{\prime}(z)\right] \geq 0 .
$$

Moreover, as the coefficients $a$ and $b$ are finite, the fulfillment of this condition also should be expected in the case when the derivatives $W^{\prime}(z)$ and $W^{\prime \prime}(z)$ are bounded and proportional to the small parameter which describes the model nonlinearity.

In general, the form of the solution obtained for the radiating star coincides with the original Vaidya-Bonnor metric and differs from it by redefinition of the charge term: $Q_{e}^{2}+Q_{t}^{2} \rightarrow \mathcal{K}$. Nevertheless, because the null-fluid current density depends on $\dot{\mathcal{K}}$, which now is a complicated function of the charges, the fluid dynamics can be different in each specific model of CNED.

The fact of proximity between the original Vaidya-Bonnor solution and its CNED analogue allows one to extend some features obtained earlier for the Vaidya-Bonnor solution on the CNED case (40). For instance, to describe thermodynamics of the star one can fully use the results obtained in [40,41], so the Hawking temperature on the event or on the cosmic horizon $r_{h}$ reads

$T_{H}=\frac{r_{h}-M-\frac{2}{3} \Lambda r_{h}^{2}-2 r_{h} \dot{r}_{h}}{2 \pi k\left(2 M r_{h}-\mathcal{K}-\frac{1}{3} \Lambda r_{h}^{4}\right)}$

where $k$ is Boltzmann constant and the horizon radius can be expressed from the null surface equation:
Table 1 Particular expressions for $\dot{\mathcal{K}}$ for some special $z$

\begin{tabular}{lll}
\hline$z=-1$ & $a_{1}=-1, b_{1}=0$ & $\dot{\mathcal{K}}=2 Q_{t} \dot{Q}_{t}\left(W-W^{\prime}\right)$ \\
& $a_{2}=0, b_{2}=0$ & \\
$z=0$ & $a_{1}=2 \sqrt{2}$, & \\
& $b_{1}=-2 \sqrt{2}$ & \\
& $a_{2}=0, b_{2}=0$, & \\
$z=1$ & $a_{1}=0, b_{1}=1$ & $\left.\dot{\mathcal{K}}=2 Q_{e}\left(W+2 \sqrt{2} W^{\prime}\right)+\dot{Q}_{t}\left(W-2 \sqrt{2} W^{\prime}\right)\right]$ \\
& $a_{2}=0, b_{2}=0$ & \\
\hline
\end{tabular}


$r_{h}^{2}-2 M r_{h}+\mathcal{K}-\frac{1}{3} \Lambda r_{h}^{4}-2 r_{h} \dot{r}_{h}=0$.

The main thermodynamic parameters, such as the entropy and the heat capacity, are usually derived from the Hawking temperature. We will not give these parameters in our paper due to their proximity to the results obtained by other authors; we note only that the analysis of thermodynamics for such a type of metric was described in detail in [41].

\section{Conclusion}

In this paper we obtained an exact analytical solutions for the compact astrophysical objects in CNED vacuum electrodynamics with the most general form of the Lagrangian.

The analog of the Reissner-Nordström solution for dyon in CNED was obtained; it needs introducing two constants with the interpretation of electric charges. In the general CNED case these constants are different; however, they coincide in Maxwell electrodynamics and in the special type of CNED proposed in Ref. (30).

It was shown that the special type of inverse black holes in CNED which possess only one thermodynamic phase transition does not agree with the fundamental restrictions coming from the causality, unitarity and the dominant energy conditions.

An extension for the Vaidya-Bonnor solution on the case of an arbitrary conformal nonlinear electrodynamics was described. It was shown that the metric functions differ from the original Vaidya-Bonnor solution only by redefinition of the charge term, which now depends on the CNED model choice. However, the dynamics of the null fluid can be sufficiently different due to the current density, which depends on the derivatives of the CNED Lagrangian, which are absent in Maxwell theory.

Data Availability Statement This manuscript has no associated data or the data will not be deposited. [Author's comment: This is theoretical work, so we have not used any data.]

Open Access This article is distributed under the terms of the Creative Commons Attribution 4.0 International License (http://creativecomm ons.org/licenses/by/4.0/), which permits unrestricted use, distribution, and reproduction in any medium, provided you give appropriate credit to the original author(s) and the source, provide a link to the Creative Commons license, and indicate if changes were made.

Funded by SCOAP . $^{3}$

\section{References}

1. R.P. Mignani, V. Testa et al., MNRAS 465, 492 (2017)

2. B. Le Garrec et al., Proc. SPIE 8962, 89620I (2014)

3. H.-P. Schlenvoigt, T. Heinzl, U. Schramm, T. Cowan, R. Sauerbrey, Physica Scripta 91, 023010 (2016)

4. A. Bashinov, A. Gonoskov, A. Kim, G. Mourou, A. Sergeev, Eur. Phys. J. Spec. Top. 223, 1105 (2014)

5. D.N. Papadopoulos et al., High Power Laser Sci. Eng. 4, E34 (2016)

6. P. Soffitta, Exp. Astron. A 144, 425 (2013)

7. M.C. Weisskopf, B. Ramsey et al., Results Phys. 6, 1179 (2016)

8. M. Born, L. Infeld, Proc. R. Soc. A 144, 425 (1934)

9. W. Heisenberg, H. Euler, Z. Phys. 26, 714 (1936)

10. E.S. Fradkin, A.A. Tseytlin, Phys. Lett. B 163, 123 (1985)

11. G.W. Gibbons, D. Rasheed, Nucl. Phys. B 454, 185 (1995)

12. V.I. Denisov, Phys. Rev. D 61, 036004 (2000)

13. B. Hofmann, L. Infeld, Phys. Rev. 51, 765 (1937)

14. S. Deser, G.W. Gibbons, Class. Quant. Grav. 15, L35 (1998)

15. S. Gangopadhyay, D. Roychowdhury, J. High Energy Phys. 2, 2012 (2012)

16. A. Sheykhi, F. Shakera, Phys. Lett. B 754, 281 (2016)

17. D. Hanneke, S. Fogwell, G. Gabrielse, Phys. Rev. Lett. 100, 120801 (2008)

18. M. Schumacher et al., Phys. Lett. B 58, 134 (1975)

19. Sh Zh Akhmadaliev, Phys. Rev. Lett. 89, 061802 (2002)

20. W.E. Lamb, R.C. Retherford, Phys. Rev. 72, 241 (1947)

21. M. Fuchs et al., Nat. Phys. 11, 964 (2015)

22. G.V. Dunne, Eur. Phys. J. D 55, 327 (2009)

23. P. Gaete, J. Helayël-Neto, Eur. Phys. J. C 74, 3182 (2014)

24. H.H. Soleng, Phys. Rev. D 52, 6178 (1995)

25. H. Salazar, A.G.D.J. Plebański, J. Math. Phys. 28, 2171 (1987)

26. S.I. Kruglov, Eur. Phys. J. C 75, 88 (2015)

27. S. Hendi, Ann. Phys. 333, 282 (2013)

28. S.I. Kruglov, Phys. Rev. D 94, 044026 (2016)

29. M. Novello, S.E. Perez Bergliaffa, J. Salim, Phys. Rev. D 69, $127301(2004)$

30. S.I. Kruglov, Phys. Rev. D 92, 123523 (2015)

31. E. Ayón-Beato, A. García, Phys. Rev. Lett. 80, 5056 (1998)

32. K.A. Bronnikov, Phys. Rev. D 63, 044005 (2001)

33. T. Fulton, F. Rohrlich, L. Witten, Rev. Mod. Phys. 34, 442 (1962)

34. M. Cataldo et al., Phys. Lett. B 484, 154 (2000)

35. J.A.R. Cembranos, A. de la Cruz-Dombriz, J. Jarillo, J. Cosmol. Astropart. Phys. 2015, 042 (2015)

36. V. Denisov, E. Dolgaya, V. Sokolov, I. Denisova, Phys. Rev. D 96, 036008 (2017)

37. A. Shabad, V. Usov, Phys. Rev. D 83, 105006 (2011)

38. S.W. Hawking, G.F.R. Ellis, The Large Scale Structure of SpaceTime (Cambridge Monographs on Mathematical Physics) (Cambridge University Press, Cambridge, 1975)

39. W.B. Bonnor, P.C. Vaidya, Gen. Relativ. Gravit. 2, 127 (1970)

40. S. Wanglin, L. Xingye, W. Li, Z. Qingxiang, Nucl. Phys. B (Proc. Suppl.) 166, 270 (2007)

41. A. Farmany, Astrophys. Space Sci. 337, 785 (2012) 